\title{
NBSIR 73-119
}

\section{LASER DAMAGE IN MATERIALS}

Albert Feldman, Deane Horowitz, and Roy M. Waxler

Solid State Materials Section

Inorganic Materials Division

Institute for Materials Research

National Bureau of Standards

Washington, D. C. 20234

February 1973

Semi-Annual Technical Report

Period Covered: August 1, 1972, to January 31, 1973

ARPA Order No. 2016

Prepared for

Advanced Research Projects Agency

Arlington, Virginia 22209 

NBSIR 73-119

\section{LASER DAMAGE IN MATERIALS}

Albert Feldman, Deane Horowitz, and Roy M. Waxler

Solid State Materials Section Inorganic Materials Division Institute for Materials Research National Bureau of Standards Washington, D. C. 20234

February 1973

Semi-Annual Technical Report

Period Covered: August 1, 1972, to January 31, 1973

\section{Prepared for}

Advanced Research Projects Agency

Arlington, Virginia 22209

ARPA Order No. 2016

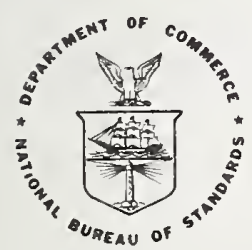

U. S. DEPARTMENT OF COMMERCE, Frederick B. Dent, Secretary NATIONAL BUREAU OF STANDARDS, Richard W. Roberts, Director 

LASER DAMAGE IN MATERIALS

\begin{abstract}
Albert Feldman, Deane Horowitz, and Roy M. Waxler
Solid State Materials Section

Inorganic Materials Division

Institute for Materials Research
\end{abstract}

\begin{abstract}
ARPA Order No. . . . . . . . 2016
Program Code Number. . . . . . . 3Dl0

Effective Date of Contract . . . . January 1, 1972

Contract Expiration Date . . . . D December 31, 1973

Principal Investigator . . . . . Albert Feldman

(301) $921-2840$
\end{abstract}

This is a progress report. The work is incomplete and is continuing. The views and conclusions contained in this document are those of the authors and should not be interpreted as necessarily representing the official policies, either expressed or implied, of the Advanced Research Projects Agency or the U. S. Government.

Parts of this report, appropriately modified, will be submitted to a professional journal for publication. 



\section{LASER DAMAGE IN MATERIALS}

\section{Abstract}

The relative contributions of the Kerr, electrostrictive, and thermal effects to the self-focusing thresholds of borosilicate crown glass, fused silica, and dense flint glass have been estimated from an analysis of damage threshold data for linearly polarized and circularly polarized radiation. The measurements were made with a Nd:glass laser operating in the TEM ${ }_{0 O}$ mode with a temporal pulse width of $25 \mathrm{~ns}$. The Kerr effect appears to be the largest effect. The thermal effect is also significant. The electrostrictive effect is smallest. Reasonable values of absorption coefficient are calculated from the thermal contribution. The results are in qualitative agreement with the work of others. Self-focusing data obtained with linearly polarized and circularly polarized radiation are presented for yttrium aluminum garnet (YAG) and five commercial Nd:doped laser glasses. The YAG data agree with the theory of self-focusing. Near the threshold the laser glass data appear to indicate intrinsic damage rather than self-focusing. Differences between the various laser glasses are small. Self-focusing data obtained in dense flint glass with a longer focal length lens are also presented. An electrooptic shutter actuated by a laser triggered spark gap is discussed. 

1. Technical Report Summary . . . . . . . . . . . 1

1.1 Technical Problem . . . . . . . . . . . . 1

1.2 General Methodology. . . . . . . . . . . . 1

1.3 Technical Results . . . . . . . . . . 2

1.4 Department of Defense Implications . . . . . . . 3

1.5 Implications for Further Research. . . . . . . . 4

2. Technical Report . . . . . . . . . . . . . 4

2.1 Self-Focusing Mechanisms in Optical Glasses. . . . 4

2.1.1 Introduction . . . . . . . . . . . . 4

2.1.2 Theoretical Considerations . . . . . . 8

2.1.2.1 The Kerr Effect. . . . . . . . . 9 9

2.1.2.2 Electrostrictive Self-Focusing . . . . . 9

2.1.2.3 Thermal Self-Focusing. . . . . . . . 12

2.1.2.4 Combined Effect. . . . . . . . . 13

2.1.3 Experimental Procedure . . . . . . . . 14

2.1.4 Results and Discussion ......... 18

2.1 .5 Conclusions. . . . . . . . . . . 26

2.2 Self-Focusing Studies in Yttrium Aluminum Garnet

(YAG), Dense Flint Glass (SF 55), and Five

Commercial Nd:Doped Laser Glasses. . . . . . 27

2.2.1 Introduction . . . . . . . . . 27

2.2.2 Self-Focusing Length Measurements. . . . 27 
2.2.3 Results and Discussion ....... 28

2.2 .3 .1 YAG . . . . . . . . 28

2.2.3.2 SF 55 Glass........... 30

2.2.3.3 Five Commercial Laser Glasses. . . . 32

2.3 Laser Triggered Spark Gap and Pockels Cell . . . 39

2.4 Acknowledgments. . . . . . . . . 39

2.5 References ............. . 4 41 
1. Technical Report Summary

1.1 Technical Problem

The main objective of this program is the study of self-focusing in materials used in conjunction with high-energy laser systems. These studies are necessary because self-focusing is the main process which leads to bulk-intrinsic damage in laser materials. The damage appears as filamentary tracks in materials exposed to high-intensity laser radiation. Three mechanisms have been proposed to explain the damage: electrostrictive, Kerr, and thermal self-focusing. An understanding of the relative importance of these mechanisms is necessary if we are to fabricate, in a systematic way, materials with high self-focusing thresholds.

\subsection{General Methodology}

Laboratory experiments were conducted to study the process of self-focusing in borosilicate crown glass (BSC 517), fused silica, dense flint glass (SF 55), yttrium aluminum garnet (YAG), and five cornmercial Nd:doped laser glasses. The output of a Q-switched Nd:glass laser operating in the TEM 00 mode was focused into the samples. The laser beam was characterized fully as to pulse energy, pulse time evolution, beam profile, and reproducibility (see section 2.1 .3 of Technical Report). Two different experiments were performed: (1) Damage thresholds were measured for both linearly and circularly polarized radiation; (2) se'lffocusing lengths, which are related to the damage track lengths, were measured as a function of laser beam peak power. 
A theoretical analysis of the data was made by assuming that the experimental damage threshold is the self-focusing threshold. The contributions of the Kerr, electrostrictive, and thermal effects to the intensity dependent refractive index were theoretically analyzed. The measurement of damage thresholds for linearly and circularly polarized radiation provided the necessary and sufficient data from which to estimate the relative importance of each of the effects to self-focusing. The selffocusing length data were compared to the theoretically predicted values.

\subsection{Technical Results}

The ratio of damage thresholds for circular polarization to linear polarization is found to be greater than unity in all the samples tested. The damage is assumed to result from self-focusing. The analysis of the data in BSC 517, fused silica, SF 55, and YAG indicates that the Kerr effect is the largest contributor to self-focusing with a significant contribution from the thermal effect. Electrostriction is the smallest of the effects for the focusing beam geometry used. The latter result contradicts an earlier report in which we neglected the thermal effect. The electrostrictive effect would be negligible for an unfocused beam. Reasonable values of the absorption coefficient are obtained from the estimated thermal effect.

Using a focused geometry with a 181-mm focal length lens, we found the self-focusing length measurements in YAG and SF 55 to be in good agreement with a theory which predicts self-focusing lengths as a function of peak power. Using a 362-mm focal length lens in SF 55, we found that the self-focusing lengths deviate from theory significantly. 
Self-focusing length measurements were made in five laser glasses with both linearly and circularly polarized radiation. The data suggest that the damage threshold measured is not the self-focusing threshold. The data deviate strongly from self-focusing theory. No significant difference was found in the behavior of the different glasses.

The capability of our laser system has been expanded to produce pulses with half-widths of $2.5 \mathrm{~ns}$. This has been accomplished by inserting a laser-triggered spark gap and electro-optic shutter into the beam. Previously, the pulse width of our laser was limited to $\sim 25 \mathrm{~ns}$.

\subsection{Department of Defense Implications}

The Department of Defense has a need for high-powered solid state laser systems. Thus it is important (1) to understand the processes which limit the output power of such systems, (2) to obtain data which suggest methods for increasing the power output of a given system, and (3) to verify theories which predict the performance of such systems. Self-focusing is the main process responsible for bulk intrinsic damage in high-power pulsed laser systems. We have addressed ourselves to the problem of self-focusing and have reached the following conclusions: (1) The Kerr effect is the main process leading to self-focusing for laser pulses with less than 25 ns width at half-maximum power. The Kerr effect increases as the refractive index increases. The implication is that materials with low refractive indices will have high self-focusing thresholds. (2) The thermal effect cannot be neglected for pulse widths $\geq 25 \mathrm{~ns}$. For shorter pulses the effect decreases in importance; however, in high repetition rate systems, thermal self-focusing can be a problem 
because of the integrating nature of the effect. Materials with lower absorption coefficients will possess higher self-focusing thresholds.

(3) Electrostriction appears to be unimportant as an effect leading to selffocusing for unfocused laser beams. (4) The current theory of self-focusing, which predicts self-focusing lengths as a function of peak power, is not always in agreement with experiment. (5) YAG has a lower self-focusing threshold than the five commercial laser glasses examined in this study.

\subsection{Implications for Further Research}

A more detailed analysis of the self-focusing data for YAG and the five laser glasses is planned. Damage threshold and self-focusing measurements are to be extended to crystalline materials, used as modulators, such as deuterated potassium dihydrogen phosphate ( $\left.K D^{*} P\right)$, lithium niobate $\left(\mathrm{IiNbO}_{3}\right)$, and calcite $\left(\mathrm{CaCO}_{3}\right)$. We also plan to measure self-focusing thresholds in materials with low refractive indices (LiF, NaF). These are expected to possess high self-focusing thresholda.

2. Technical Report

\subsection{Self-Focusing Mechanisms in Optical Glasses}

2.1.1 Introduction

The output of high-powered laser systems is limited by the damage occurring in components exposed to the intense electromagnetic radiation. Self-focusing [1] is the main process leading to bulk intrinsic damage in the components of o-switched solid state laser systems. An under standing of the mechanisms underlying self-focusing is necessary if we are ever to fabricate in a systematic manner materials with high self- 
focusing thresholds. For example, it has been proposed that electrostrictive effects can be largely eliminated by the production of materials with extremely small electrostrictive coefficients [2] .

The mechanism responsible for the self-focusing of laser beams with pulse widths of $\sim 25 \mathrm{~ns}$ has not been uniquely identified. Three mechanisms have been proposed to explain the effect: electrostriction $[3-7]$, Kerr effect $[3-5,8]$, and thermal self-focusing [9-12]. Up to the present time insufficient data exists to judge the comparative importance of the proposed mechanisms. Self-focusing in liquids is known to be caused by the Kerr effect because of the large contribution of freely rotatable molecules or ions to the nonlinear index coefficient $\mathrm{n}_{2}$. Because solids lack freely rotatable molecules, it was thought that electrostriction [3-5] was the dominant mechanism. Duguay and Hansen have calculated the nonlinear index of refraction due to the Kerr effect $n_{2}(K)$ from measurements of induced birefringence in borosilicate crown glass using picosecond pulses $[13,14]$. They find that the value obtained is of sufficient magnitude to cause self-focusing and are of the opinion that the Kerr effect is the predominant mechanism for all pulse widths.

E. L. Kerr [7] has developed the theory of electrostrictive self-focusing to explain the data of steinberg [15], who used 55-ns pulses from a ruby laser to measure damage thresholds in several optical glasses. Kerr is of the view that electrostriction is the principal self-focusing mechanism for pulse widths $z 10$ ns. For pulses less than 
$10 \mathrm{~ns}$, on the other hand, the electrostrictive mechanism has insufficient time to take effect, and thus will decrease in magnitude as the pulse length decreases.

For glasses such as we are considering, thermal self-focusing was not thought to be important. Quelle [12], in a sample calculation, showed that the threshold for thermal self-focusing would be two orders of magnitude greater than for electrostriction. However, other authors [16] indicated that the thermal effect would be of comparable magnitude.

In this paper we present a semiquantitative estimate of the relative contributions of the Kerr, electrostrictive, and thermal effects to self-focusing in borosilicate crown glass (BSC 517), fused silica, and dense flint glass (SF 55) from measurements of self-focusing thresholds for circular and linear polarization $[17,18]$. We believe this to be the first attempt to take into account all three effects. The method was suggested in an earlier letter [17] in which we showed that the damage threshold for circular polarization was higher than for linear polarization. In the letter, we had deduced the relative contributions of the kerr effect and electrostriction to selffocusing assuming the thermal effect could be neglected. In this work we find a significant thermal contribution. The Kerr effect appears to be the largest of the effects. Electrostriction is found to be the smallest, in disagreement with E. I. Kerr. Kerr used the Clausius-Mosotti (Iorentz-Iorenz) relations to calculate the electrostrictive index change In order to explain self-focusing on the basis of electrostriction alone, 
whereas we have calculated the electrostrictive index change using the experimentally determined stress-optic coefficients. The change of refractive index with density calculated with the Clausius-Mosotti relation agrees poorly with experiment when applied to solids [19].

In Section 2.1.2 we discuss the theoretical framework upon which our estimates are based. We include an analysis of electrostrictive strains and of thermally induced strains that takes into account the constraining effect of the medium surrounding the irradiated portion of the sample. We also calculate the expected ratios of self-focusing threshold for circular and linear polarization for each of the effects acting alone.

In Section 2.1.3 we discuss the experimental procedure and the data we use in our calculations. We have obtained the data by focusing into the samples the output of a Q-switched Nd:glass laser ( $\tau=25 \mathrm{~ns}$ ) operating in the $\mathrm{TEM}_{\mathrm{O}}$ mode. These data are thought to be more indicative of the self-focusing threshold than earlier measurements [17] because a longer focal length lens was used. Zverev and Pashkov [16] have pointed out the importance of using a long focal length lens for measuring self-focusing thresholds.

In Section 2.1 .4 we calculate the total nonlinear index $\mathrm{n}_{2}$ from the threshold data and the contribution of electrostriction $n_{2}(E S)$, the Kerr effect $n_{2}(K)$, and the thermal effect $n_{2}(T)$ to $n_{2}$. The integrating nature of the thermal effect is taken into account in these calculations. The numbers obtained for $\mathrm{n}_{2}$ are in good agreement with values calculated by owyoung et al. [20] from elliptical polarization rotation measurements. We also estimate the absorption coefficients necessary to account for $n_{2}(T)$ and obtain reasonable values. 


\subsubsection{Theoretical Considerations}

Self-focusing of laser radiation occurs when the focusing effect due to the intensity-dependent refractive index exceeds the spreading of the beam by diffraction. The refractive index of the medium can be expressed in the form

$$
n=n_{0}+n_{2} E^{2}
$$

where $E$ is the RMS electric field obtained from an average over several optical cycles. The second order nonlinear index $n_{2}$ is a function of time unless it derives from mechanisms that instantaneously follow the temporal shape of the laser pulse. For the experimental conditions in this paper, both the Kerr and the electrostrictive effects are instantaneous, but the thermal effect is never instantaneous. Thus, in general, a critical power for self-focusing will not exist. For a given pulse shape, $E^{2}=E_{0}^{2} f(t)$, we can write Eq. (1) in the form

$$
\delta n=n_{2}(t) E_{0}^{2},
$$

where $E_{0}^{2}$ is the peak value of $E^{2}$, and the time dependence of $E^{2}$ is incorporated into $n_{2}(t)$. At a time $t=t_{0}, \delta n$ will have a maximum value. At a critical value of $E_{0}^{2}$, self-focusing will take place and we can obtain an expression for the critical peak pulse power $P_{t}$ necessary to cause self-focusing. It must be emphasized that the maximum index change will. not occur at the peak of the pulse. The expression for $P_{t}$ that we use has been derived for beams with a Gaussian profile $[7,16,21]$ :

$$
P_{t}=\lambda^{2} c /\left(32 \pi^{2} n_{2}\right)
$$

where $\lambda$ is the wavelength of the laser radiation in air, $c$ is the velocity of light, and $n_{2}$ has been redefined to equal $n_{2}\left(t_{0}\right)$. Equation (3) is also equal to $P_{1}$ in the work of Dawes and Marburger [22]. 
2.1.2.1. The Kerr Effect

In this work we assume that the Kerr effect arises from nonlinearities in the electronic polarizabilities and that orientational effects can be ignored. Recent experimental work [20] suggests this is indeed the case. Thus the Kerr effect would be instantaneous up to optical frequencies.

For an isotropic material the nonlinear polarization is given by [23]

$$
\overrightarrow{\mathrm{P}}_{\mathrm{NL}}=3 \mathrm{c}_{1122}(\overrightarrow{\mathrm{E}} \cdot \overrightarrow{\mathrm{E}}) \overrightarrow{\mathrm{E}}
$$

$c_{1122}$ is a constant. If we insert the field for a linearly polarized wave

$$
\vec{E}=E_{0}[\cos (\omega t-k z), 0,0]
$$

and a circularly polarized wave of equal intensity

$$
\vec{E}=E_{0}[\cos (\omega t-k z), \sin (\omega t-k z), 0] / \sqrt{2}
$$

into Eq. (4) and calculate the Fourier component of $\overrightarrow{\mathrm{P}}_{\mathrm{NL}}$ at frequency $\omega$, we obtain a ratio of 1.5 for $n_{2}(K) / n_{2}^{\prime}(K)$. Throughout this paper primed symbols refer to circular polarization, and unprimed symbols to linear polarization. Third harmonic contributions can be ignored because the phase matching condition is not met.

\subsubsection{Electrostrictive Self-Focusing}

Electrostrictive self-focusing is due to refractive index changes caused by strains induced by the strong electric fields present in high-powered Iaser radiation. E. L. Kerr has treated the full dynamical problem of electrostrictive self-focusing [7]. He has shown that the electrostrictive response will depend on the parameter $x=a_{0} / v \tau$, 
where $a_{0}$ is the $1 / e$ intensity point radius of a Gaussian beam at the focus of a lens in the absence of self-focusing, $v$ is the longitudinal sound velocity in the medium, and $\tau$ is the temporal pulse width of the laser beam at half the maximum power. Kerr has shown that for $x<1$, the electrostrictive strain has time to develop fully and hence $\mathrm{n}_{2}$ (ES) can be calculated from the steady state. For our experimental conditions, $a_{0}=14.7 \mu \mathrm{m}, \tau=25 \mathrm{~ns}$, and $\mathrm{v}$ varies from $3 \times 10^{3} \mathrm{~m} / \mathrm{s}$ to $5 \times 10^{3} \mathrm{~m} / \mathrm{s}$, yielding $x=0.1$ to 0.2 . The electrostriction can follow the pulse shape and hence the instantaneous approximation is valid. Kerr, in treating the problem of electrostrictive selffocusing, has ignored the tensorial nature of the effect because of the complexity of solving vectorial differential equations. In view of the steady state nature of the problem as applied to our work, we treat the effect as a statics problem and therefore we can take into account the tensorial properties of electrostriction. We calculate $n_{2}$ (ES) and $\mathrm{n}_{2}^{\prime}(\mathrm{ES})$ for a material containing a uniform electric field in a cylindrical region and zero electric field outside this region. A rigorous calculation would be more complex due to the Gaussian spatial variation of the laser field. However, the solution we obtain is rigorously correct on the axis of the laser beam.

The strain induced in the cylindrical region due to the intense electric field is given by [24]

$$
\varepsilon_{k l}=\gamma_{i j k l} E_{i} E_{j} / 2+s_{k l m n}{ }^{p} m
$$

where $\gamma$ is the electrostriction tensor, $S$ is the elastic compliance 
tensor, and $\mathrm{P}$ is the stress tensor. The stress comes from the constraining effect of the field free region. From a set of thermodynamical Maxwell's equations, we can show that [25-27]

$$
\gamma_{i j k l}=-k_{i m}{ }^{k} q_{m n k l} / 4 \pi,
$$

where $k$ is the dielectric tensor and $q$ is the stress-optic tensor.

The change in dielectric tensor with strain is given by

$$
\Delta\left[k^{-1}\right]_{i j}=p_{i j k l} \varepsilon_{k l} ;
$$

$p$ is the elasto-optic tensor. In order to calculate $n_{2}$ (ES) and $n_{2}$ (ES), we must first calculate the electrostrictive strains

$$
\varepsilon_{k l}^{0}=\gamma_{i j k l} E_{i} E_{j} / 2
$$

For an isotropic material the net strain becomes [28]

$$
\begin{aligned}
& \varepsilon_{11}=\left[2\left(\varepsilon_{11}^{\circ}+\varepsilon_{22}^{0}\right)+(3-4 \nu)\left(\varepsilon_{11}^{0}-\varepsilon_{22}^{\circ}\right)\right] /[8(1-\nu)] ; \\
& \varepsilon_{22}=\left[2\left(\varepsilon_{11}^{\circ}+\varepsilon_{22}^{0}\right)-(3-4 \nu)\left(\varepsilon_{11}^{\circ}-\varepsilon_{22}^{0}\right)\right] /[8(1-\nu)] ; \\
& \varepsilon_{33}=0
\end{aligned}
$$

where $v$ is the Poisson ratio. These strain components are inserted into Eq. (9) and the final result is:

$$
\begin{aligned}
& n_{2}^{\prime}(E S)=n_{0}^{7}\left(q_{11}+q_{12}+2 v q_{12}\right)\left(p_{11}+p_{12}\right) /[64(1-v)] \\
& n_{2}(E S)=n_{0}^{7}\left(q_{11}-q_{12}\right)\left(p_{11}-p_{12}\right)(3-4 v) /[128(1-v)]+n_{2}^{\prime}(E S)
\end{aligned}
$$

Equation (12) is identically equal to Kerr's [7] steady state limit. 


\subsubsection{Thermal self-Focusing}

Thermal self-focusing results from positive refractive index

changes caused by absorptive heating of a sample exposed to high-energy laser radiation. For an isotropic material the change of index with temperature is

$$
\frac{d n}{d T}=\left(\frac{\partial n}{\partial T}\right)-\frac{n_{0}^{3}(1+v)\left(p_{11}+p_{12}\right) \beta}{12(1-v)}
$$

$\beta$ is the volume coefficient of expansion. We assume that heat diffusion does not occur during the passage of the laser pulse. The thermal effect differs from the two previous effects in that it depends upon the integrated energy of the pulse.

The first term in Eq. (14), the change of index with temperature at constant density, follows the instantaneous temperature. The change in temperature is governed by electron-phonon relaxation times which are fast compared to $25 \mathrm{~ns}$. The term is positive in most glasses.

The second term in Eq. (14) is the change in index with temperature due to thermoelastic strains. It is derived with the same boundary conditions used to compute the electrostrictive strain. A similar term has been derived by zverev et al. [29], who have pointed out that, due to the constraining stresses, thermal self-focusing can take place even though the conventional value of $\mathrm{dn} / \mathrm{dT}<0$. The response time of the thermoelastic strain term is equal to the response time of electrostriction. Since $\mathrm{x}<\mathrm{I}$ it will also respond to the temperature 
instantaneously. The change of index due to the thermal effect is [9]

$$
\delta n(t)=\frac{\alpha c n_{0}}{4 \pi C_{p} \rho} \frac{d n}{d T} E_{0}^{2} \int_{-\infty}^{t} f\left(t_{1}\right) d t_{1}
$$

where $\alpha$ is the absorption coefficient, $c$ is the velocity of light, $C_{P}$ is the specific heat at constant pressure, and $\rho$ is the density. We assume that the absorption coefficient is small so that the laser beam undergoes insignificant attenuation while passing through the sample. From Eqs. (3) and (15) we see that the thermal self-focusing threshold will vary inversely with the pulse width for a given peak power. There will be no polarization effects for isotropic materials, i.e., $n_{2}(T) / n_{2}(T)=1$.

\subsubsection{Combined Effect}

The change of index will equal the sum of the contributions from each of the effects. At this point it becomes convenient to assume that $f(t)$ has a Gaussian shape, i.e.,

$$
f(t)=\exp \left[-4 t^{2} / \tau^{2}\right] \text {, }
$$

where $\tau_{e} \approx 1.2 \tau$. The change of index is then

$$
\delta n=\left[n_{2}(K)+n_{2}(E S)\right] E_{0}^{2} \exp \left[-4 t^{2} / \tau_{e}^{2}\right]+n_{2}(T) E_{0}^{2}\left[1+\operatorname{erf}\left(2 t / \tau_{e}\right)\right]
$$

where

$$
n_{2}(T)=\frac{.075}{\sqrt{\pi}} \frac{\alpha n_{0} \tau}{c_{P} \rho} \frac{d n}{d T}
$$

and $\operatorname{erf}(x)$ is the error function. The maximum value of $\delta \mathrm{n}$ occurs at times 


$$
\begin{aligned}
& t_{0}=\tau e^{n_{2}(T) / 2 \sqrt{\pi}\left[n_{2}(E S)+n_{2}(K)\right] ;} \\
& t_{0}^{\prime}=\tau e^{n_{2}}(T) / 2 \sqrt{\pi}\left[n_{2}^{\prime}(E S)+2 n_{2}(K) / 3\right] .
\end{aligned}
$$

The nonlinear index is then

$$
\begin{aligned}
& n_{2}=\left[n_{2}(K)+n_{2}(E S)\right] \exp \left[-\left(2 t_{0} / \tau_{e}\right)^{2}\right]+n_{2}(T)\left[1+\operatorname{erf}\left(2 t_{0} / \tau_{e}\right)\right] \\
& n_{2}^{\prime}=\left[n_{2}^{\prime}(K)+n_{2}^{\prime}(E S)\right] \exp \left[-\left(2 t_{0}^{\prime} / \tau_{e}\right)^{2}\right]+n_{2}(T)\left[1+\operatorname{erf}\left(2 t_{0}^{\prime} / \tau_{e}\right)\right] .
\end{aligned}
$$

\subsubsection{Experimental Procedure}

The experimental apparatus is shown in Fig. 1 [30]. It contains a Q-switched Nd:glass laser oscillator operating in the TEM mode. The output of the oscillator has been characterized as to energy, temporal pulse width, peak power, and beam profile.

The energy was measured by a thermopile and found to be constant to within $\pm 2 \%$. The temporal pulse shape was monitored by a high-speed photodiode and oscilloscope. The pulse width at half the maximum power was $25 \mathrm{~ns}$. The peak power was obtained by setting the pulse energy equal to the integral of the pulse shape over time.

The beam profile of the laser beam is obtained at the focusing lens position by measuring the diameter of burn patterns on unexposed developed Polaroid [31] film. The output of the laser is maintained at constant pulse energy but is attenuated from pulse to pulse by interposing calibrated attenuation filters between the oscillator and the film. The burn pattern radius is plotted as a function of beam energy and fitted to a Gaussian curve, $\mathrm{H}=\mathrm{H}_{0} \exp \left(-\mathrm{r}^{2} / \mathrm{a}^{2}\right)$ where the 


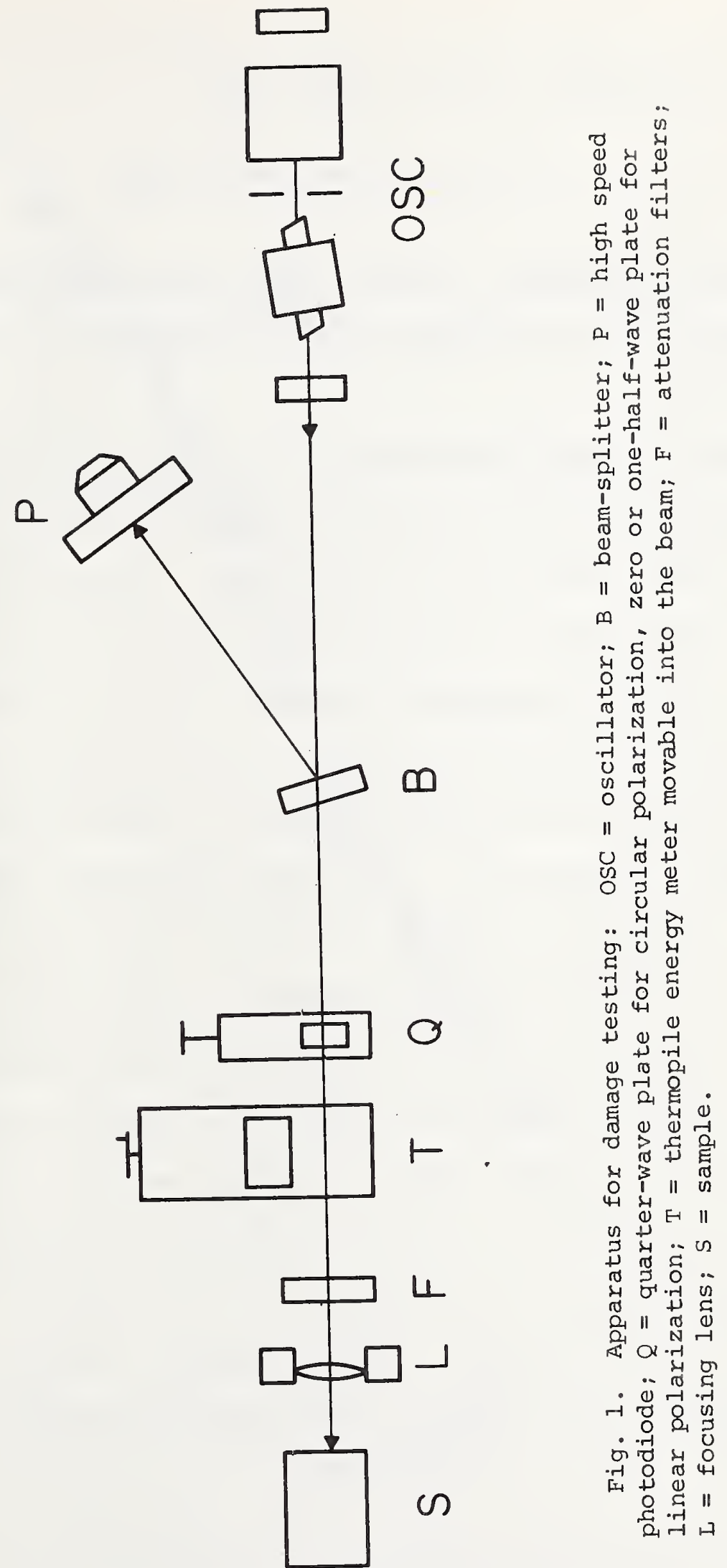


constant $\mathrm{H}_{0}$ is obtained in units of $\mathrm{J} / \mathrm{cm}^{2}$ from

$$
w=2 \pi H_{0} \int_{0}^{\infty} \exp \left(-r^{2} / a^{2}\right) r a r ;
$$

$W$ is the total pulse energy. Figure 2 shows a plot of the beam profile together with the Gaussian which is fitted by eye. The pulse energy is $99 \mathrm{~mJ}$.

Damage threshold measurements for linearly and circularly polarized radiation $[17,18]$ were made by focusing the laser beam into the samples using a lens of $181 \mathrm{~mm}$ focal length. The oscillator output was linearly polarized in the horizontal plane with the ratio of horizontal to vertical polarization greater than 200:1. Circularly polarized light was produced by inserting a quarter-wave plate into the beam. The quarter-wave plate consisted of a fused silica plate under uniaxial compression [32]. The linear polarization damage measurements were made by relaxing the stress in the plate. Measurements were also made with the plate stressed to a half-wave retardation; the results were consistent with the unstressed plate measurements.

The damage thresholds were obtained by observing whether or not damage occurred in the sample during any particular laser shot. Each laser shot was focused onto a different position in the sample. The energy of the laser pulse was attenuated in $\sim 20 \%$ increments with calibrated attenuation filters. Finer increments of energy were ohtained ly varying the oscillator pump energy. The criterion for damage was the presence of a fractured region in the vicinity of the 


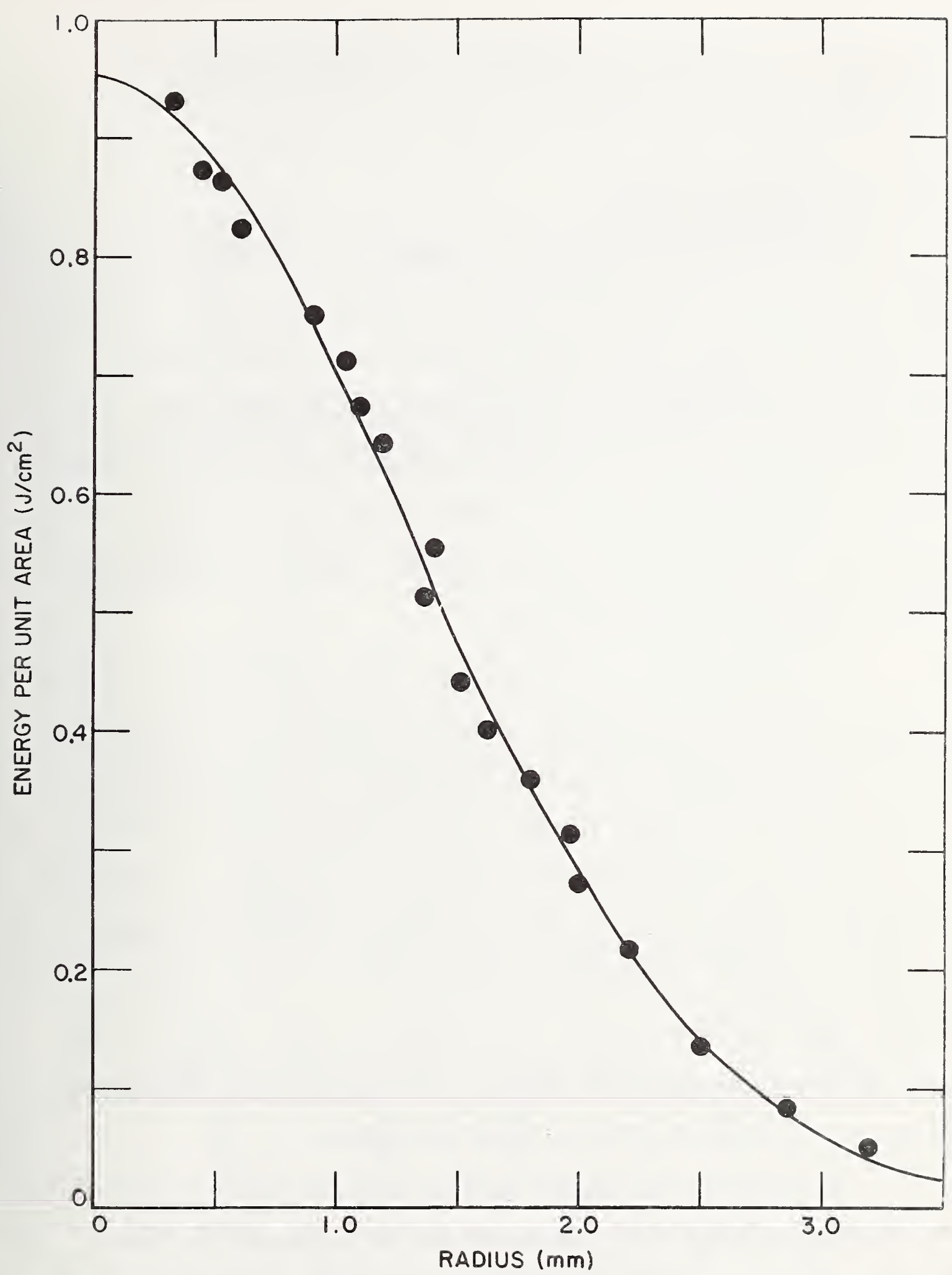

Fig. 2. Beam Profile. Energy per unit area as a function of distance from laser beam axis as measured at the focusing lens. The solid line is a Gaussian fit to the data. 
focal spot. In all cases, a bright flash of visible radiation was emitted when damage took place.

\subsubsection{Results and Discussion}

Damage threshold measurements have been made in borosilicate crown glass (BSC 517), fused silica, and dense flint glass (SF 55). The data are shown in Fig. 3. Solid points represent pulses that produced damage; open points represent pulses that did not produce damage. The scatter in the data is due to sample variability, laser energy variations and the statistical nature of the actual damage process $[33,34]$. In all cases the threshold for circularly polarized light $\mathrm{p}_{t}$ has been higher than for linearly polarized light $P_{t}$.

We now proceed to calculate $n_{2}, n_{2}(E S), n_{2}(K)$, and $n_{2}(T)$ under the assumption that the damage results from self-focusing. All the necessary input parameters for the calculations are given in Table I. The total nonlinear indices $n_{2}$ and $n_{2}^{\prime}$ are calculated from $P_{t}$ and $P_{t}^{\prime}$ using Eq. (3). We calculate $n_{2}$ (ES) and $n_{2}^{\prime}$ (ES) with Eqs. (12) and (13). The numbers for $\mathrm{p}_{11}, \mathrm{p}_{12}, \mathrm{q}_{11}$, and $\mathrm{q}_{12}$ given in Table $\mathrm{I}$ have been measured at visible wavelengths; however, we do not expect them to differ significantly at $1.06 \mathrm{~m}$. The values for $\mathrm{n}_{2}(\mathrm{~K})$ and $\mathrm{n}_{2}(\mathrm{~T})$ are calculated by numerically solving Eqs. (19) and (20) with the values for $n_{2}, n_{2}^{\prime}, n_{2}$ (ES) and $n_{2}^{\prime}(E S)$ as input parameters.

We summarize our results in Table II. The limits of error arise from the scatter in the experimental data. An examination of the results indicates that the kerr effect is the largest contributor to 
Table I. Input Parameters Necessary for Calculating the Contributions to Self-Focusing.

BSC 517 Fused Silica Dense Flint (SF 55)

\begin{tabular}{|c|c|c|c|}
\hline$c_{p}\left(10^{6} \in r g g^{-1} K^{-1}\right)$ & $8.6^{a}$ & $7.5^{b}$ & $8 \cdot 4^{C}$ \\
\hline$\rho\left(\mathrm{g} \mathrm{cm}^{-3}\right)$ & $2.51^{\mathrm{a}}$ & 2.2 & $4.72^{c}$ \\
\hline$(\partial n / \partial T)_{\rho}\left(10^{-6} K^{-1}\right)$ & $8.3^{\mathrm{d}}$ & $9.7^{\mathrm{e}}$ & $21.5^{\mathrm{d}}$ \\
\hline$\beta\left(10^{-6} \mathrm{~K}^{-1}\right)$ & $20.0^{a}$ & 1.5 & $24.6^{c}$ \\
\hline$q_{11}\left(10^{-13} \mathrm{~cm}^{2} /\right.$ dyn $)$ & $0.315^{f}$ & $0.418^{9}$ & $1.7^{\mathrm{h}}$ \\
\hline$q_{12}\left(10^{-13} \mathrm{~cm}^{2} / \mathrm{dyn}\right)$ & $1.92^{\mathrm{F}}$ & $2.71^{9}$ & $2.2^{h}$ \\
\hline$p_{11}$ & $0.115^{f}$ & $0.120^{9}$ & $0.21^{h}$ \\
\hline$p_{12}$ & $0.221^{f}$ & $0.270^{9}$ & $0.23^{h}$ \\
\hline$v$ & $0.204^{a}$ & 0.164 & $0.247^{c}$ \\
\hline$n_{0}(1.06 \mu \mathrm{m})$ & 1.507 & 1.450 & 1.733 \\
\hline
\end{tabular}

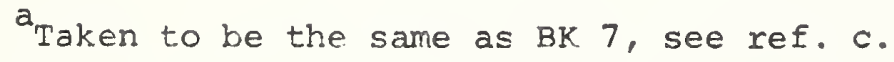

b. B. Sosman, The Properties of Silica (Chemical Catalogue company, ljew York, 1927), p. 314 .

${ }^{C}$ Catalogue, Schott Optical Glass, Inc., Duryea, Pennsylvania.

${ }^{d}$ Calculated from $\mathrm{dn} / \mathrm{dT}$ given in ref. $\mathrm{c}$ and from the elasto-optic coefficients. 
Table I. (continued)

${ }^{e}$ Calculated from $\mathrm{dn} / \mathrm{dT}$ given in J. B. Austin, "A Vacuum Apparatus of Measuring Thermal Expansion at Elevated Temperatures, with Measurements on Platinum, Gold, Magnesium, and Zinc," Physics, vol. 3, pp. 240-267 (1932), p. 248, and from the elasto-optic coefficients.

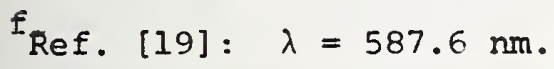

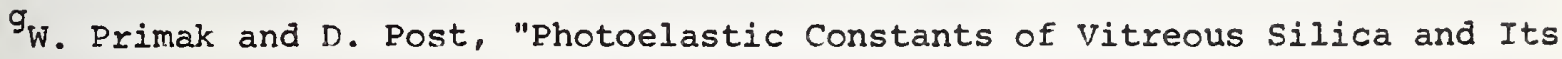
Elastic Coefficient of Refractive Index," J. Appl. Phys., vol. 30, pp. 779-788 (1959): $\lambda=546.1 \mathrm{~nm}$.

$\mathrm{h}_{\text {Ref. [17]. }}$ 
Table II. Contributions of Kerr, Electrostrictive, and Thermal Effects to Self-Focusing Thresholds. ${ }^{a}$

$\begin{array}{lll}\text { BSC } 517 & \text { Fused Silica } & \text { Dense Flint SF } 55 \\ 0.88 \pm .05 & 1.17 \pm .06 & 0.155 \pm .007 \\ 1.10 \pm .07 & 1.45 \pm .07 & 0.205 \pm .007 \\ 1.21 \pm .07 & 0.92 \pm .05 & 6.9 \pm .3 \\ 0.97 \pm .07 & 0.74 \pm .05 & 5.2 \pm .2 \\ 0.13 & 0.16 & 0.68 \\ 0.11 & 0.13 & 0.68 \\ 0.72 \pm .22 & 0.49 \pm .15 & 5.2 \pm 0.7 \\ 0.33 \pm .22 & 0.24 \pm .15 & 1.0 \pm 0.7 \\ (3 \pm 2) \times 10^{-3} & (9 \pm 6) \times 10^{-4} & (5 \pm 4) \times 10^{-3}\end{array}$

$a_{\text {The errors denote the scatter in the data. }}$ 
self-focusing for the beam parameters of this experiment. The data indicate that the electrostrictive effect is much smaller than the Kerr effect. This conclusion differs considerably from our earlier resuit [17] because in that work we had neglected two important effects in addition to making a factor of two error in the equations for $n_{2}$ (ES) and $n_{2}^{\prime}(E S)$. (An appropriate correction for this error was made in ref. [18].) In our earlier paper, we had ignored the thermal effect and we had ignored the constraining effects of the medium surrounding the irradiated portion of the sample when calculating the electrostrictive effect. Additionally, the data upon which we base our calculations here have been taken with a longer focal length lens than the earlier work [17] and thus more accurately indicate the selffocusing threshold. The importance of using a long focal length lens for self-focusing measurements has been pointed out by zverev and Pashkov [16]. They showed that the threshold measured with a short focal length lens would be more indicative of the intrinsic damage threshold, because for a given pulse power, the energy density would be higher at the focal spot.

Our results indicate that the thermal effect is of considerable importance for self-focusing with $25 \mathrm{~ns}$ pulses. Because of the pulse width dependence of $\mathrm{n}_{2}(\mathrm{~T})$, the importance of the thermal effect for shorter pulses decreases. However, because of its integrating nature, thermal self-focusing can be very important in high repetition rate systems even though the individual pulses are quite short. It is of interest to deduce the absorption coefficient necessary to account for 
$n_{2}(T)$. These values are presented at the bottom of Table II. The numbers obtained are consistent with parameters for optical glasses. Our value of $\alpha$ for BSC 517 is close to the value for a similar giass, BK $7\left(\alpha=3 \times 10^{-3} \mathrm{~cm}^{-1}\right)$, as reported by McMahon [35]. The absorption coefficient for our fused silica is significantly larger than the largest value measured by Rich and Pinnow $\left(\alpha=1.6 \times 10^{-4} \mathrm{~cm}^{-1}\right)$ [36]. There are several explanations for the discrepancy. First, the fused silica we used may actually have a higher absorption coefficient. Second, our assumption that the damage thresholds we measure are the self-focusing thresholds may not be valid. The threshold values, especially the value for circular polarization, are of such a magnitude that they may be more indicative of the intrinsic damage threshold than of a self-focusing threshold. The result would be to make the ratio $\mathrm{P}_{t}^{\prime} / \mathrm{P}_{t}$ come closer to unity, resulting in an apparently larger thermal effect. It is most likely that some self-focusing is taking place since, in the absence of self-focusing, we would expect $\mathrm{P}_{t}^{\prime} / \mathrm{P}_{t}=1$.

A comparison of our results with the data of other workers is shown in Table III. Owyoung et al. [20] have calculated $\mathrm{n}_{2}$ from the rotation of the principal axes of elliptically polarized light from a single frequency ruby laser. Their data agree quite favorably with ours. The differences observed could be explained by dispersion effects, by differences in the glasses, and by corrections to Eq. (3). We justify the use of Eq. (3) instead of $\mathrm{P}_{2}$ of ref. [22] for two reasons: The size of the thermal effect would be increased by a factor $\approx 4$, yielding larger absorption coefficients than seems reasonable; the 
Table III. Comparison of Nonlinear Index Coefficients in Units of $10^{-13}$ esu.
$\mathrm{n}_{2} \underline{a}$
$n_{2}$ b/
$n_{2}(K) \stackrel{b}{-}$

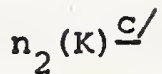
$n_{2}(K) \stackrel{d /}{-}$

Fused silica

1.2

0.92

0.49

$\mathrm{BK}-7$

1.7

0.6

1.0

BSC 517

1.2

0.72

SF 7

6.9

SF 55

6.9

5.2

LaSF 7

3

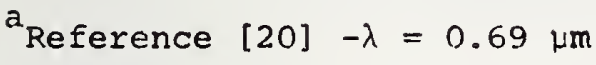

$\mathrm{b}_{\text {This work }}-\lambda=1.06 \mu \mathrm{m}$

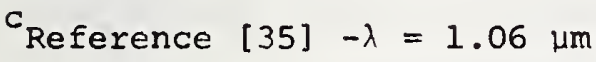

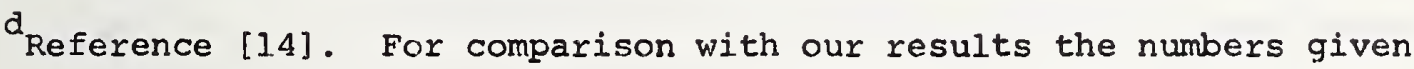
are half the values in the reference because of the differences in the methods used. 
results we obtain using $\mathrm{Eq}$. (3) give better agreement with the work of others. The excellent agreement for the flint glasses (SF 55 and SF 7) is probably fortuitous.

Our value of $\mathrm{n}_{2}(\mathrm{~K})$ in BSC 517 agrees quite well with the value obtained by McMahon [35] from self-focusing measurements with picosecond pulses in BK 7. The agreement is also good compared to the value from Duguay and Hansen [14]. Their value for LasF 7, a dense flint glass, agrees with our value for SF 55 flint glass within a factor of two. Duguay and Hansen measure $n_{2}(K)$ from the intensity induced birefringence caused by picosecond pulse. Because of the different method of measurement, the numbers given in Table III are half the values in the reference [14].

From our calculations we note that $n_{2}(K)$ increases with refractive index. This is in qualitative agreement with the work of Wang [37].

\subsubsection{Conclusions}

We have estimated the relative contributions of the Kerr effect, electrostriction, and thermal effects to the self-focusing thresholds of borosilicate crown glass, fused silica and dense flint glass from an analysis of damage threshold data for linearly polarized and circularly polarized radiation. For the beam geometry used, we conclude that the Kerr effect is the largest effect in all the samples tested. The thermal effect is also found to be significant, but the electrostrictive effect is quite small. Both of the latter effects are expected to be insignificant for an individual subnanosecond pulse: 
however, because of its integrating nature, thermal self-focusing can be important in high repetition rate laser systems.

The results we have obtained are in qualitative agreement with the work of others. Absorption coefficients calculated from the estimated thermal contribution to self-focusing appear to be reasonable for commercial optical glasses. It would be of interest to measure the absorption coefficient directly for a comparison with the value obtained from the self-focusing measurements.

2.2. Self-Focusing Studies in Yttrium Aluminum Garnet (YAG), Dense Flint Glass (SF 55), and Five Commercial Nd:Doped Laser Glasses.

\subsubsection{Introduction}

In this section of the technical report we present self-focusing data on YAG, SF 55 glass, and five commercial laser glasses. We have measured self-focusing lengths as a function of peak laser power using both linearly and circularly polarized radiation. In all cases the circularly polarized radiation had a higher damage threshold than the linearly polarized radiation. To our knowledge, the self-focusing measurements in YAG are the first to be reported.

2.2.2. Self-Focusing Length Measurements

Recent experiments $[33,38-41]$ have shown that the filamentary damage observed when solids are exposed to high-intensity laser radiation is due to self-focusing of the laser beam and that the self-focused spot moves toward the laser source as the pulse power rises. Assuming that the nonlinear index coefficient $\mathrm{n}_{2}$ responds instantaneously to the laser 
pulse shape, Dawes and Marburger [22] have made a detailed computer calculation which predicts the self-focusing length as a function of beam power. We have produced damage filaments in optical glasses by focusing the laser beam at different power levels $P$ into samples using $181 \mathrm{~mm}$ and $362 \mathrm{~mm}$ focal length lenses. The experimental arrangement is the same as reported in section 2.1 .3 . We have measured a length $\mathrm{z}_{\mathrm{f}}$ from the upstream end of the damage track to the sample entrance face and than have calculated the self-focusing length $z$ from the formula [41]

$$
z^{-1}=z_{f}^{-1}-R^{-1}
$$

where $R$ is the radius of curvature of the laser wavefront at the sample entrance face. $R$ is calculated from the measured focal point of the lens and the propagation characteristics of Gaussian beams. The normalized inverse self-focusing length $\left(z^{*}\right)^{-1}$ is plotted as a function of $\mathrm{p}^{1 / 2}$.

$$
\begin{aligned}
& z^{\star}=z / 2 k a^{2} ; \\
& k=2 \pi n_{0} / \lambda,
\end{aligned}
$$

where $a$ is the $I / e$ intensity point radius of a Gaussian beam, $n_{0}$ is the index of refraction, and $\lambda$ is the wavelength $(1.06 \mu \mathrm{m})$. 2.2.3. Results and Discussion $2.2 .3 .1 . Y A G$

Figure 4 shows a plot of $\left(z^{*}\right)^{-1}$ vs. $P^{1 / 2}$ for undoped YAG. The square data points have been obtained with linearly polarized radiation; 


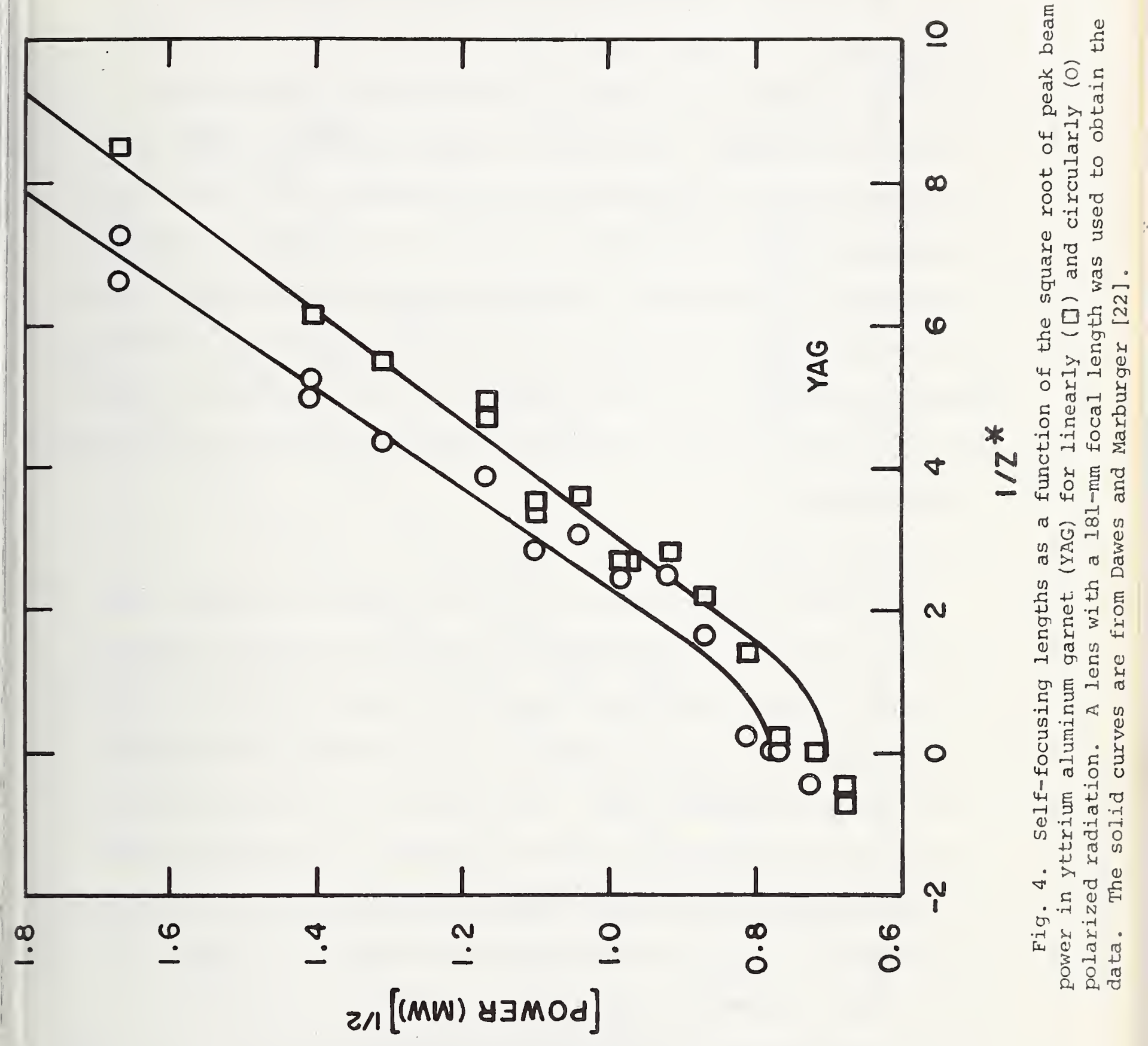


the circular points have been obtained with circularly polarized radiation. The data have been obtained by focusing the laser beam into the sample with a $181 \mathrm{~mm}$ focal length lens along the [111] crystallographic axis. Also plotted in the figure are the theoretical curves derived by Dawes and Marburger. The curves are fitted empirically to the data by varying only the critical power for self-focusing. The values that we obtain are $\mathrm{P}_{t}=0.49 \mathrm{MW}$ and $\mathrm{P}_{t}^{\prime}=0.61 \mathrm{MW}$. The agreement of theory and experiment is very good. A preliminary analysis of the data indicates that the Kerr effect is the main self-focusing mechanism with a sizable thermal contribution. The electrostrictive effect is negligible because YAG has exceedingly small stress-optic coefficients compared to the glasses we have measured.

\subsubsection{SF 55 Glass}

Figure 5 shows a plot of $\left(z^{*}\right)^{-1}$ vs $\mathrm{P}^{1 / 2}$ for SF 55 glass. The square data points have been obtained with linearly polarized radiation; the circular points have been obtained with circularly polarized radiation. The data have been obtained by focusing the laser beam into the sample with a $362 \mathrm{~mm}$ focal length lens. Also plotted in the figure are the theoretical curves derived by Dawes and Marburger. The critical power for self-focusing is obtained by empirically extrapolating the data to the point $\left(z^{*}\right)^{-1}=0$. The values that we obtain are $P_{t}=0.30 \mathrm{MW}$ and $P_{t}^{\prime}=0.41 \mathrm{MW}$. Agreement between theory and the data points is poor. Earlier self-focusing measurements in SF $55 \mathrm{glass,} \mathrm{in} \mathrm{which} \mathrm{a} 181 \mathrm{~mm}$ focal length lens was used, have given better agreement with theory [18]. 


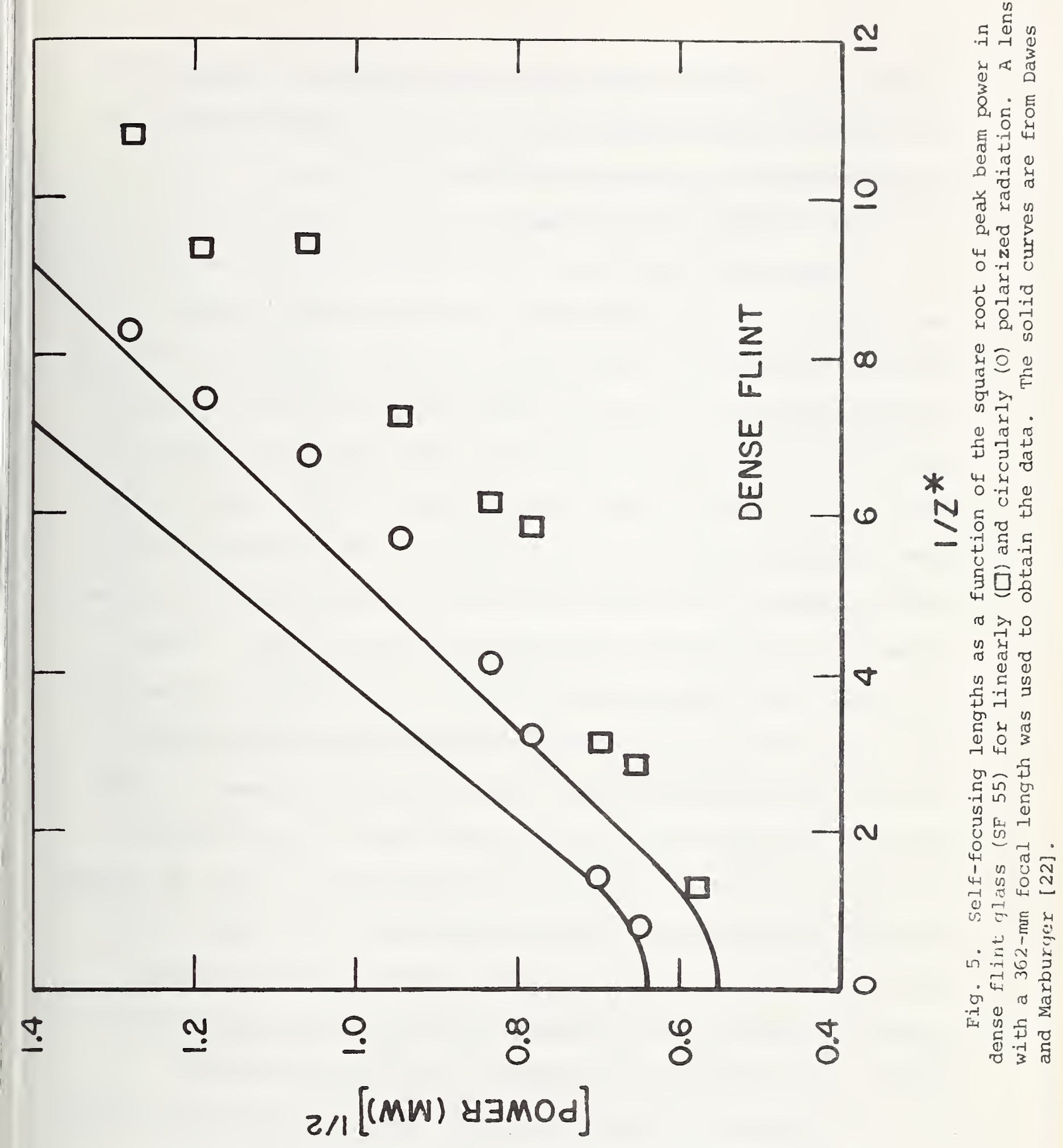


In addition, the critical power values obtained with the 362 mm lens are about twice the values obtained with the $181 \mathrm{~mm}$ lens (see Table II). We have not resolved the discrepancy at present.

2.2.3.3. Five Commercial Laser Glasses

Figures $6 a-6 e$ are plots of $\left(z^{*}\right)^{-1}$ vs $\mathrm{p}^{1 / 2}$ for five commercial laser glasses. The plus points have been obtained with linearly polarized radiation; the circular points have been obtained with circularly polarized radiation. The data have been obtained by focusing the laser beam into the samples with a $181 \mathrm{~mm}$ focal length lens. No attempt was made to fit the theory of Dawes and Marburger to the data because of the gross discrepancy between theory and experiment. The experimental selffocusing lengths are much shorter than the values predicted by the theory. The behavior of the different laser glasses is very similar. The data to the left of the figures appear to follow a curve which is concave downward. This is the qualitative type of behavior that would be expected for an intrinsic damage threshold with no self-focusing present. To the right of the figures the data follow a curve which is concave upward. This type of behavior is typical of self-focusing data. In all the glasses, the lowest damage point for circular polarization occurs closer to the laser source than the next more powerful laser shot. It is uncertain at this time whether this is a physical effect or just an experimental artifact. The effect is not as apparent with linear polarization. A comparison of the different laser glasses suggests that glasses $A, D$, and $E$ have about the same damage and self-focusing thresholds. Glasses $B$ and $C$ have about the same thresholds, but the values are slightly lower than ir. $F_{2}, D_{1}$, and $E$. 
Figs. $6 a-6 e$. Self-focusing lengths as a function of the square root of peak beam power in five commercial laser glasses for linearly (+) and circularly (O) polarized radiation. A lens with a $181-\mathrm{mm}$ focal length was used to obtain the data. 


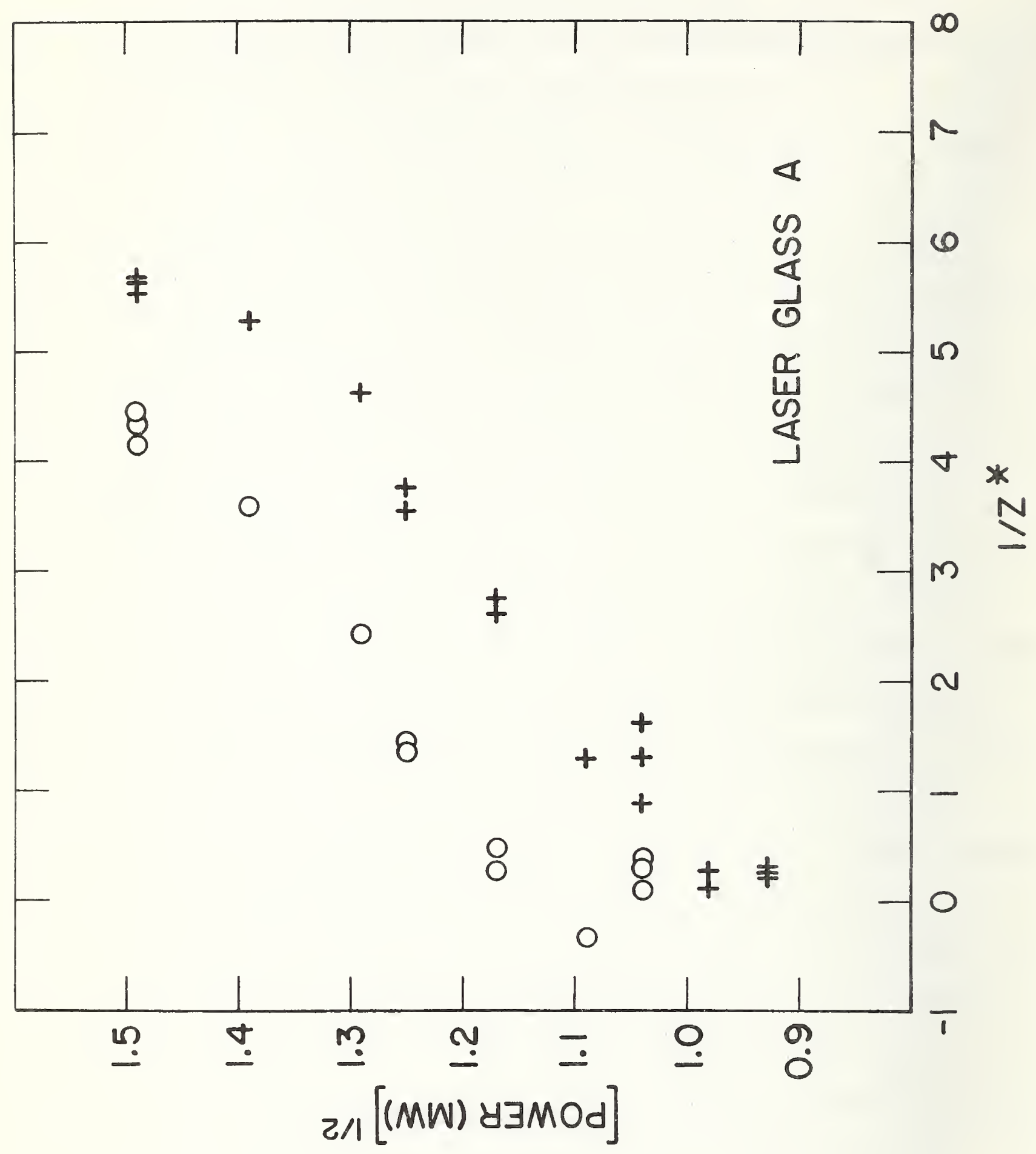




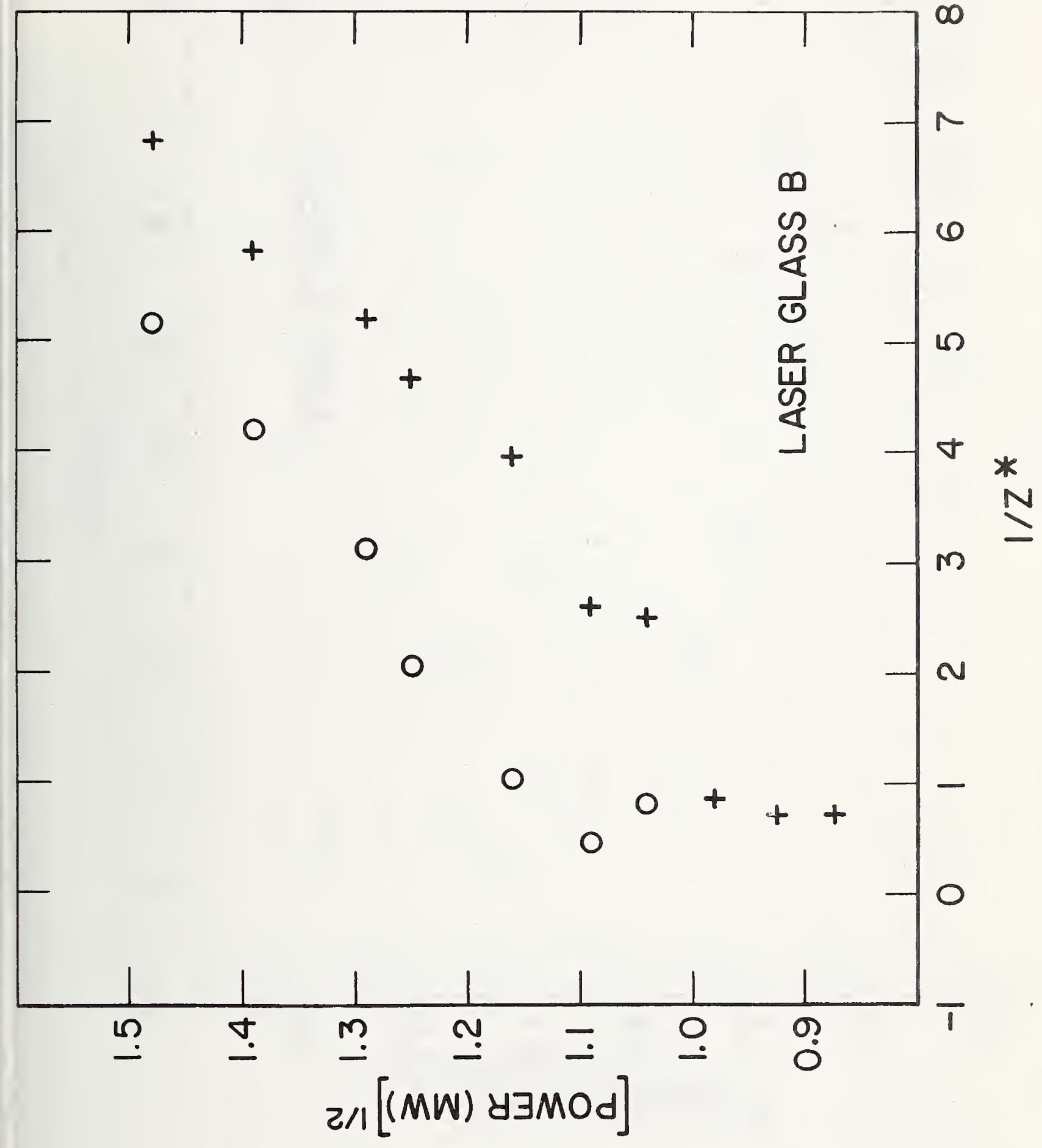




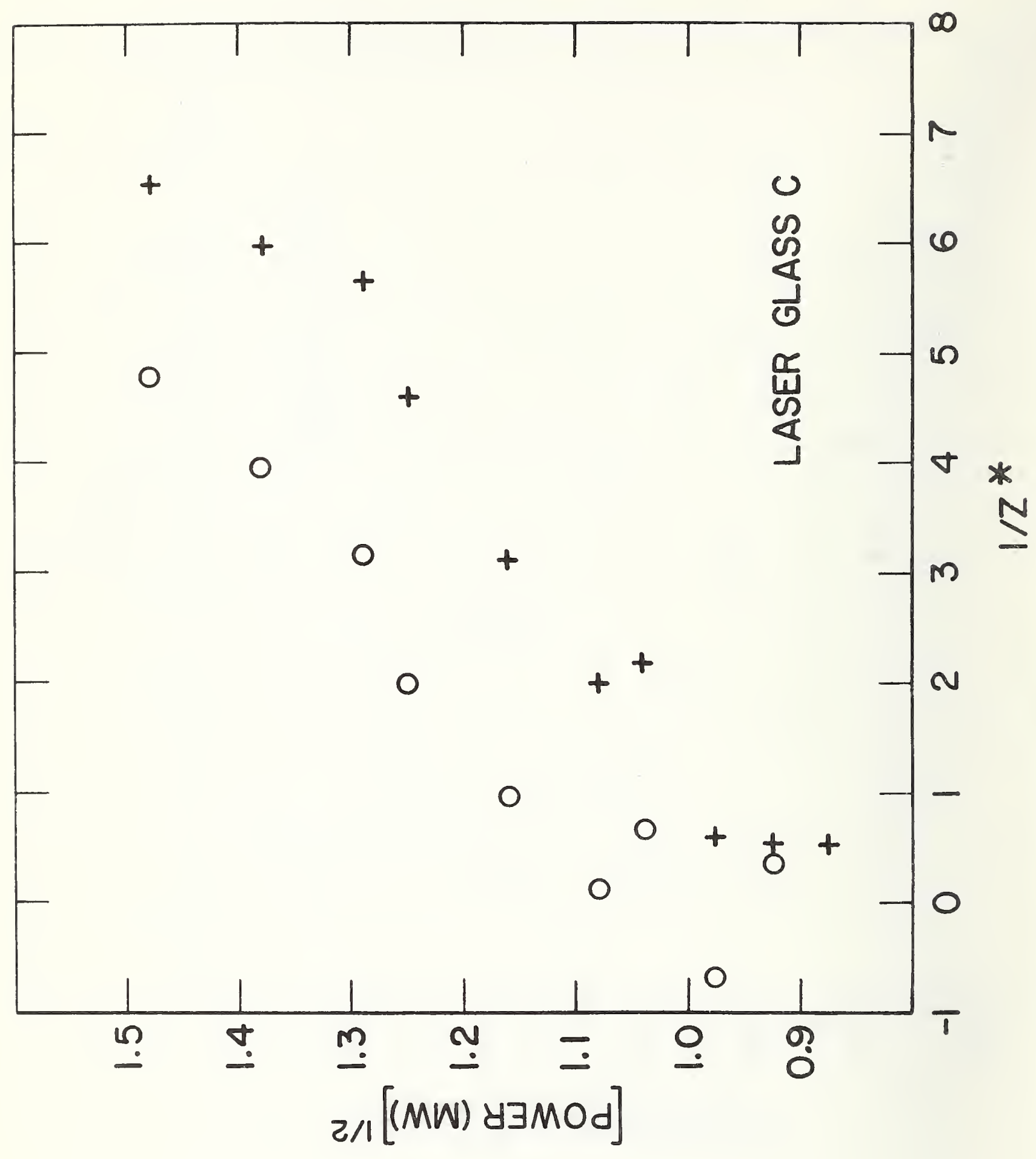




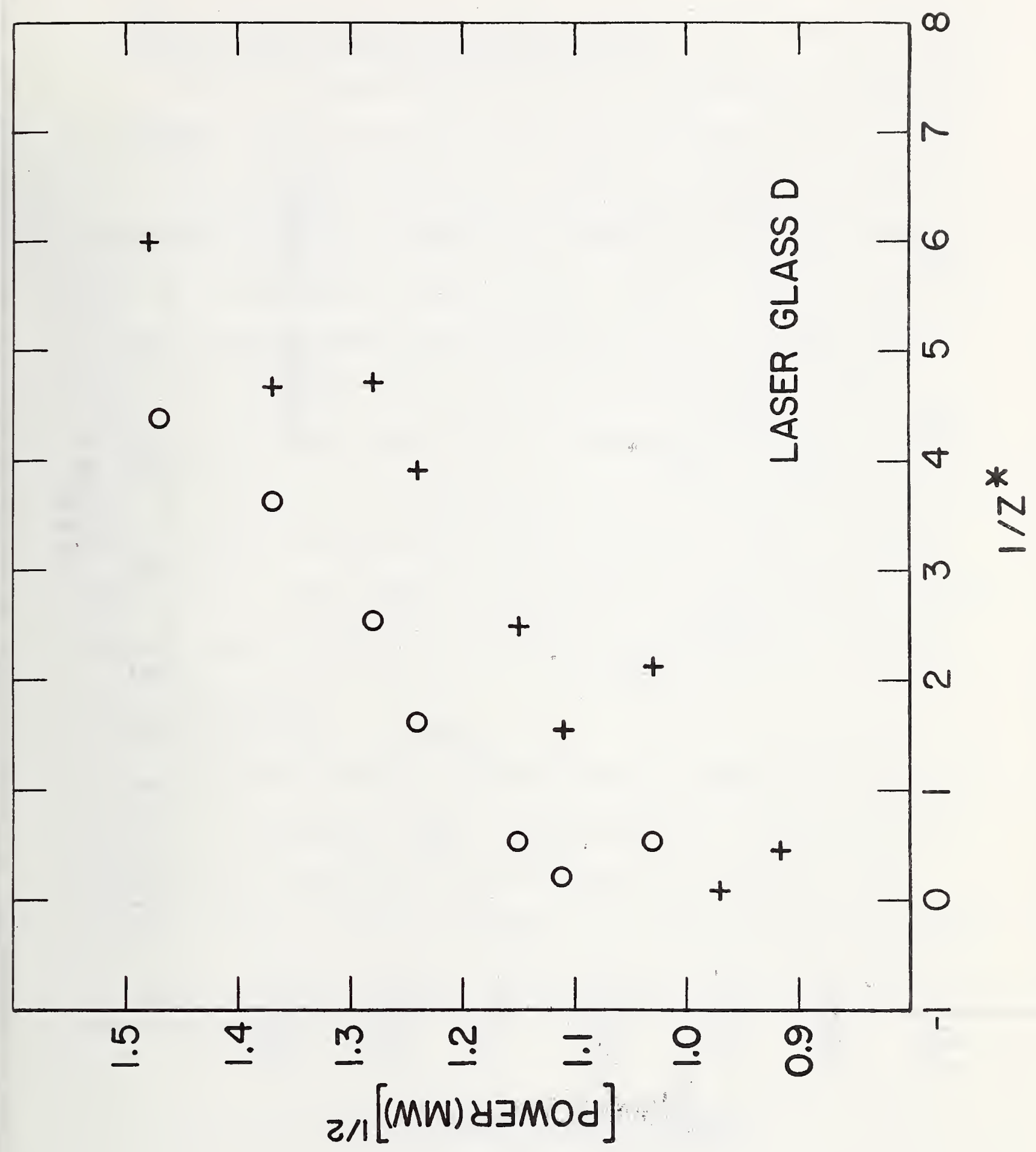

$\underset{ن}{0}$ 


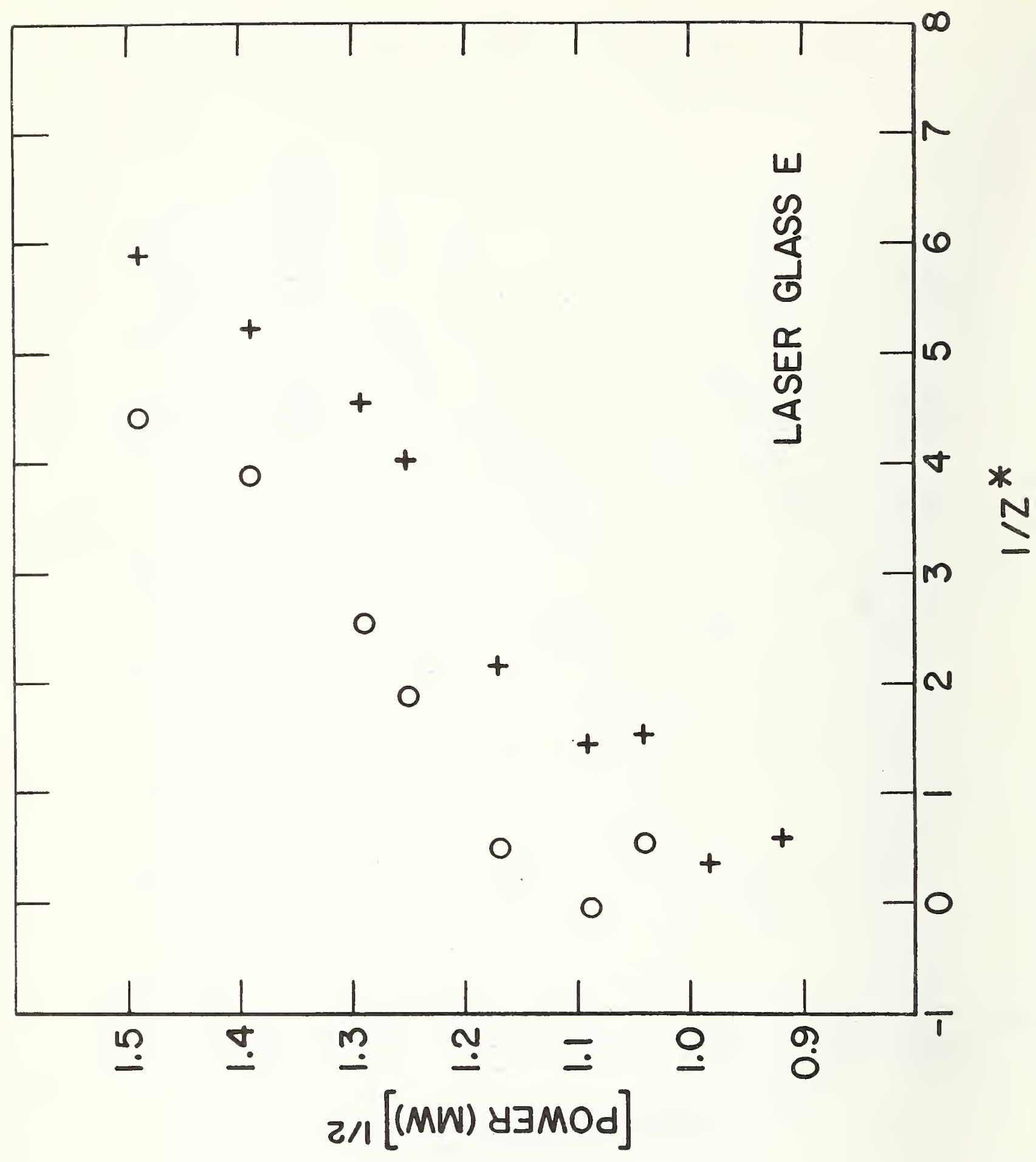




\subsection{Laser Triggered Spark Gap and Pockels Cell}

A laser triggered spark gap has been constructed for producing a short high-voltage electrical pulse in synchronization with the laser pulse. When the electrical pulse is applied to a Pockels cell with an appropriate time delay, we are able to extract from our laser pulse, a pulse with a much shorter temporal width. Figure 7 shows the effect of the laser triggered spark gap and Pockels cell upon our laser pulse. Figure $7 \mathrm{a}$ is a tracing from an oscilloscope depicting the temporal evolution of the laser pulse directly from the laser source. Figure $7 \mathrm{~b}$ depicts the portion of the pulse removed by the spark gap and Pockels cell. Figure $7 \mathrm{c}$ shows the transmitted pulse whose width at half the maximum power is $2.5 \mathrm{~ns}$. This apparatus will permit us to make damage and selffocusing studies with shorter pulses than we had available previously.

\subsection{Acknowledgments}

We thank Roland De Wit of the National Bureau of standards for his help in calculating the electrostrictive strains. We also thank Norman Boling of Owens-Illinois Glass Company, Erlan Bliss of the Lawrence Livermore Laboratories, Edwin Kerr of the Perkin-Elmer Corporation, and $A$. Owyoung of Sandia Corporation for valuable discussions concerning this work. 
a

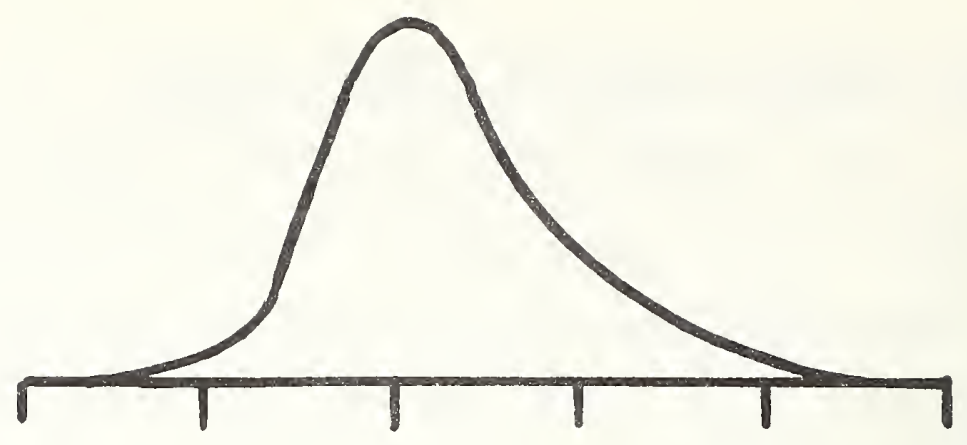

20 ns/div

b

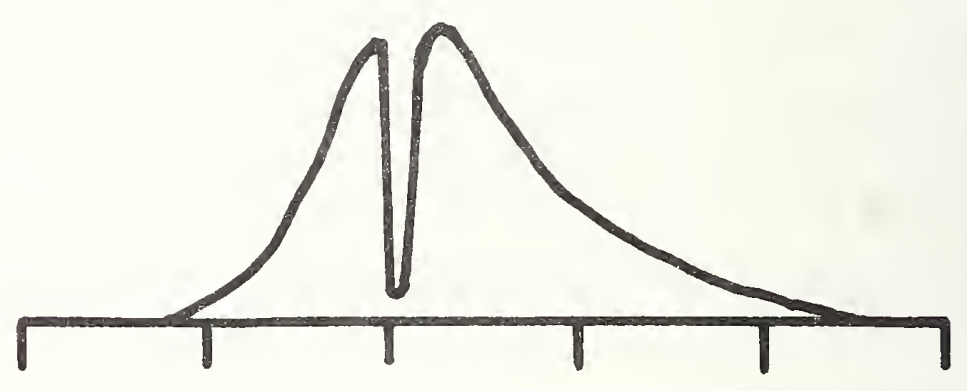

20 ns/div

C

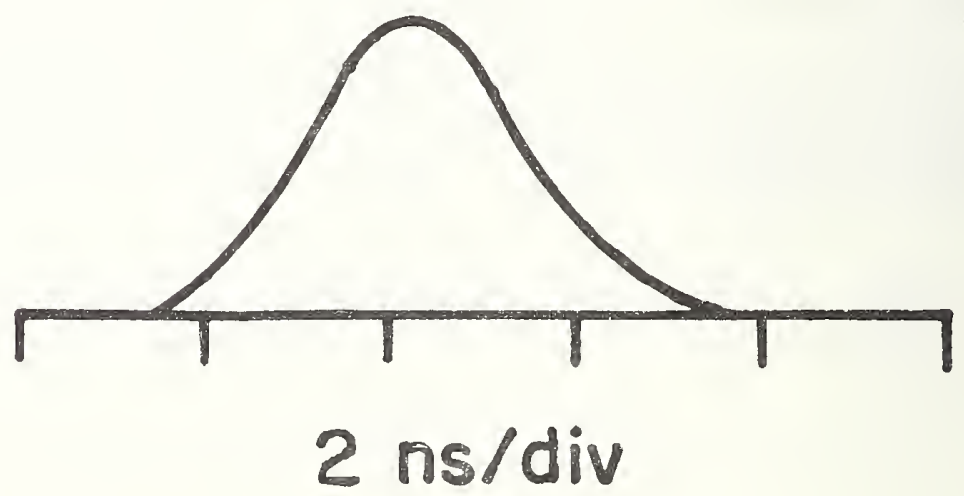

Fig. 7. Effect of laser-triggered spark gap and Pockels cell upon laser temporal pulse shape. (a) Pulse from oscillator. (b) Portion of pulse removed from oscillator pulse. (c) Transmitted pulse. 


\subsection{References}

[1] S. P. Akhmanov, A. P. Sukhorukov, and R. V. Khokhlov, "SelfFocusing and Diffraction of Light in a Nonlinear Medium," Usp. Fiz. Nauk vol. 93, pp. 19-70 (1967) [Soviet Phys.--Usp., vol. 93, pp. 609-636 (1968)] .

[2] R. M. Waxler, "Laser Glass Composition and the Possibility of Eliminating Electrostrictive Effects," IEEE J. Quantum Electronics, vol. QE-7, pp. 166-167 (1971).

[3] R. Y. Chiao, E. Garmire, and C. H. Townes, "Self-Trapping of Optical Beams," Phys. Rev. Letters, vol. 13, pp. 479-482 (1964).

[4] Ya. B. Zel'dovich and Yu. P. Raizer, "Self-Focusing of Light. Role of Kerr Effect and Striction," Zh. Eksperim. i Teor. Fiz. Pis'ma Redaktsiyu, vol. 3, pp. 137-141 (1966) [Soviet Phys. JETP Letters, vol. 3, pp. 86-89 (1966)].

[5] Y. R. Shen, "Electrostriction, Optical Kerr Effect and SelfFocusing of Iaser Beams," Phys. Rev. Letters, vol. 20, pp. 378-380 (1966) .

[6] A. A. Chaban, "Self-Focusing of Light in Solids Via the Electrostriction Mechanism," Zh. Eksperim. i Teor. Fiz. Pis'ma Redaktsiyu, vol. 6, pp. 487-490 (1967) [Soviet Phys. JETP Letters, vol.6, pp. 20-23 (1967)] .

[7] E. L. Kerr, "Track Formation in Optical Glass Caused by Electrostrictive Laser Beam Self-Focusing," Phys. Rev. A, vol. 4, pp. $1195-1218$ (1971). 
[8] A. A. Chaban, "Self-Focusing of Light in the Kerr Fiffect," Zh. Eksperim. i. Teor. Fiz. Pis'ma Redaktsiyu, vol. 5, pp. 61-64 (1967) [Soviet Phys. -- JETP Letters, vol. 5, pp. 48-51 (1967)].

[9] A. G. Litvak, "Self-Focusing of Powerful light Beams by Thermal Effects," zh. Eksperim. i Teor. Fiz. Pis'ma Redaktsiyu, vol. 4, pp. 341-345 (1966) [Soviet Phys. -- JETP Letters, vol. 4, pp. 230-233, (1966)] .

[10] Yu. P. Raiser, "Self-Focusing of a Homogeneous Light Beam in a Transparent Medium Due to Weak Absorption," Zh. Eksperim. i Teor. Fiz. Pis'ma Redaktsiyu, vol. 4, pp. 124-128 (1966) (Soviet Phys. -JETE Letters, vol. 4 , pp. 85-88 (1966)] .

[11] Yu. P. Raiser, "Self-Focusing and Defocusing, Instability and Stabilization of Light Beams in Weakly Absorbing Media," Zh. Fksperim. i teor. Fiz. Pis'ma Redaktsiyu, vol. 52, pp. 470-482 (1967) [Soviet Phys. -- JETP, vol. 25, pp. 308-316 (1967)].

[12] F. W. Quelle, "Self-Focusing in Glass," in Damage in Laser Materials, ASTM STP 469, American Society for Testing and Materials, 1969, pp. $110-116$

[13] M. A. Duguay, J. W. Hansen, and S. I. Shapiro, "Study of Nd:Glass Laser Radiation," IEEE J. Quantum Electronics, vol. QE-6, pp. 725743 (1970).

[14] M. A. Duguay and J. W. Hansen, "Measurement of the Nonlinear Index $n_{2}$ of Glass Using Picosecond Pulses," in Damage in Laser Materials (NBS Special Publication 341), pp. 45-50. 
[15] G. N. Steinberg, "Filamentary Tracks Formed in Transparent Optical Glass by Laser Beam Self-Focusing. I. Experimental Investigation," Phys. Rev. A, vol. 4, pp. 1182-1194 (1971).

[16] G. M. Zverev and V. A. Pashkov, "Self-Focusing of Laser Radiation in Solid Dielectrics," Zh. Eksperim. i Teor. Fiz. Pis'ma Redaktsiyu, vol. 57, pp. 1128-1138 [Soviet Phys. -- JETP, vol. 30, pp. 616-621 (1970)] .

[17] A. Feldman, D. Horowitz, and R. M. Waxler, "Relative Importance of Electrostriction and the Kerr Effect to Self-Focusing in Optical Glasses," Appl. Phys. Letters, vol. 21, pp. 260-262 (1972).

[18] A. Feldman, D. Horowitz, and R. M. Waxler, "Relative Contribution of Kerr Effect and Electrostriction to Self-Focusing," in Laser Induced Damage in Optical Materials: 1972, NBS Special Publication 372 (U.S. Government Printing Office, Washington, D. C. 20402, 1972) pp. 92-99.

[19] R. M. Waxler and C. E. Weir, "Effect of Hydrostatic Pressure on the Refractive Indices of Some Solids," J. Res. Natl. Bur. Standards, vol. A69, pp. 325-333 (1965).

[20] A. Owyoung, R. W. Hellwarth, and N. George, "Intensity-Induced Changes in Optical Polarizations in Glasses," Phys. Rev. B, vol. 5 , pp. $628-633(1972)$.

[21] W. G. Wagner, H. A. Haus, and J. H. Marburger, "Large-Scale SelfTrapping of Optical Beams in the Paraxial Ray Approximation," Phys. Rev., vol. 175, pp. 256-266 (1968). 
[22] E. I. Dawes and J. H. Marburger, "Computer Studies in Self-Focusing," Phys. Rev., vol. 179, pp. 862-868 (1969).

[23] P. D. Maker and R. W. Terhune, "Study of optical Effects Due to an Induced Polarization Third Order in the Electric Field Strength," Phys. Rev., vol. 137, pp. A801-A818 (1965).

[24] J. F. Nye, in Physical Properties of Crystals, Coxford University Press, London, 1957), p. 257.

[25] H. Osterberg and J.W. Cookson, "The Piezodielectric Effect and Electrostriction in Anisotropic or Isotropic Media," Phys. Rev., vol. 51, pp. 1096-1101 (1937).

[26] P. W. Forsbergh, Ir., "Piezoelectricity, Electrostriction and Ferroelectricity," in Encyclopedia of Physics Vol. XVII, Dielectrics (Springer Verlag, Berlin, 1956), edited by S. Flugge, p. 360.

[27] A. A. Maradudin and E. Burstein, "Relation Between Photoelasticity, Electrostriction, and First Order Raman Effect in Crystals of the Diamond Structure," Phys. Rev., vol. 164, pp. 1081-1099 (1967).

[28] M. A. Jaswon and R. D. Bhargava, "Two-Dimensional Elastic Inclusion Problems," Proc. Camb. Phil. Soc., vol. 57, pp. 669-680 (1961).

[29] G. M. Zverev, E. A. Levchuk, E. K. Maldutis, and V. A. Pashkov, "Thermal Self-Focusing of Laser Radiation in Media with Negative dn/dT," Zh. Eksperim. i Teor. Fiz. Pis'ma Redaktsiyu, vol. 11, pp. 177-181 (1970) [Soviet Phys. -- JETP Letters, vol. 11, pp. 108-111 (1.970)

[30] A. Feldman, R. Waxler, and D. Horowitz, "Laser Induced Damage Studies," in NBS Technical Note 703, ARPA-NBS Program of Research 
on High Temperature Materials and Laser Materials, edited by A. D. Franklin and H. S. Bennett, pp. 12-36.

[31] Certain commercial equipment, instruments, or materials are identified in this paper in order to adequately specify the experimental procedure. In no case does such identification imply recommendation or endorsement by the National Bureau of Standards, nor does it imply that the material or equipment identified is necessarily the best available for the purpose.

[32] R. M. Waxler and E. N. Farabaugh, "Photoelastic Constants of Ruby," J. Res. Natl. Bur. Standards, vol. 74A, pp. 215-220 (1970) .

[33] M. Bass and H. H. Barrett, "The Probability and Dynamics of Damaging Optical Material with Lasers," in Damage in Laser Materials: 1971, NBS Special Publication 356 (U.S. Government Printing Office, Washington, D. C., 1971), pp. 76-90.

[34] M. Bass and H. H. Barrett, "Laser-Induced Damage Probability at $1.06 \mu \mathrm{m}$ and $0.69 \mu \mathrm{m}, "$ in Laser Induced Damage in Optical Materials: 1972, NBS Special Publication 372 (U.S. Government Printing office, Washington, D. C., 1972), pp. 58-69.

[35] John M. McMahon, "Damage Measurements with Subnanosecond Pulses," in Laser Induced Damage in Optical Materials: 1972, NBS Special Publication 372 (U.S. Government Printing office, Washington, D. C., 1972), pp. 100-103.

[36] T. C. Rich and D. A. Pinnow, "Total Optical Attenuation in Bulk Fused Silica," Appl. Phys. Letters, vol. 20, pp. 264-266 (1972). 
[37] Charles C. Wang, "Empirical Relation Between the Linear and the ThirdOrder Nonlinear Optical Susceptibilities," Phys. Rev. B, vol. 2, pp. 2045-2048 (1970) .

[38] G. M. Zverev, E. K. Maldutis and V. A. Pashkov, "Development of SelfFocusing Filaments in Solid Dielectrics," Zh. Eksperim. i Teor. Fiz. Pis'ma Redaktsiyu, vol. 9, p. 108 (1969) [Soviet Phys. -- JETP Letters, vol. 9, p. 61 (1969)].

[39] C. R. Giuliano, "Time Evolution of Damage Tracks in Sapphire and Ruby," in Damage in Laser Materials: 1971, NBS Special Publication 356 (U. S. Government Printing office, Washington, D. C., 1971), p. 44 .

[40] C. R. Giuliano and J. H. Marburger, "Observations of Moving Self-Foci in Sapphire," Phys. Rev. Letters, vol. 27, p. 905 (1971).

[41] J. Marburger, "Theory of Self-Focusing for Fast Nonlinear Response," in Damage in Laser Materials: 1971, NBS Special Publication 356 (U. S. Government Printing Office, Washington, D. C., 1971), p. 51 . 
FORM NBS.T IAA (1.71)

\begin{tabular}{|c|c|c|}
\hline $\begin{array}{c}\text { U.S. DEPT. OF COMM. } \\
\text { BIBLIOGRAPHIC DATA } \\
\text { SHEET }\end{array}$ & $\begin{array}{c}\text { 1. PUBLICATION OR REPORT NO. } \\
\text { NBSIR 73-119 }\end{array}$ & $\begin{array}{c}\text { 2. GOV't Accession } \\
\text { No. }\end{array}$ \\
\hline 4. TITLE AND SUBTITLE & & \\
\hline
\end{tabular}

Laser Damage in Materials

5. Publication Date

February 1973

6. Performing Otganization Code

NBSIR 73-119

7. AUTHOR(S) Albert Felcman,

Deane Horcwitz, and Roy M. Waxler

9. PERFORMING ORGANIZATION NAME AND ADDRESS

NAT IONAL BUREAU OF STANDARDS

DEP ARTMENT OF COMMERCE

WASHINGTON, D.C. 20234

8. Performing Organization

10. Project/Task/Work Unit No.

3130440

11. Contract/Grant No.

$2016 / 3010$

12. Sponsoring Organization Name and Address

Advanced Research Projects Agency

ARIINGTON, Virginia 22209

13. Type of Report \& Period

Covered Semi-Annual Tech.

8-1-72 to $1-31-73$

14. Sponsoring Agency Coje

15. SUPPLEMENTARY NOTES

16. ABSTRACT (A 200-sord or less factual summary of most significant information. If document includes a signific ant bibliography or iiterature survey, mention it here.)

The relative contributions of the Kerr, electrostrictive, and thermal effects to the self-focusing thresholds of borosilicate crown glass, fused silica, and dense flint glass have been estimated from an analysis of damage threshold data for linearly polarized and circularly polarized radiation. The measurements were made with a Nd:glass laser operating in the TEMoo mode with a temporal pulse width of $25 \mathrm{~ns}$. The Kerr effect appears to be the largest effect. The thermal effect is also significant. The electrostrictive effect is smallest. Reasonable values of absorption coefficient are calculated from the thermal contribution. The results are in qualitative agreement with the work of others. Self-focusing data obtained with linearly polarized and circularly poiarized radiation are presented for yttrium aluminum garnet (VAG) and five commercial Na:doped laser glasses. The YAG data agree with the theory of self-focusing. Near the threshold the laser glass data appear to indicate intrinsic damage rather than self-focusing. Differences between the various laser glasses are small. Self-focusing data obtained in dense flint glass with a longer focal length lens are also presented. An electro-optic shutter actuated by a laser triggered spark gap is discussed.

17. KEY H'ORDS (Alphabeticai order, separated by semicoions) Absorption coefficient; damage threshold; electrostrictive self-focusing; electrostriction; Kerr effect; laser damage; nonlinear index of refraction; self-focusing; thermal seif-focusing.

18. AVAILABILITY STATEMENT

UNLIMITED.

FOR OFFICIAL DISTRIBUTION. DO NOT RELEASE TO NTIS.

\begin{tabular}{|l|c|}
$\begin{array}{l}\text { 19. SECURITY CLASS } \\
\text { (THIS REPORT) }\end{array}$ & 21. NO. OF PAGES \\
UNCL ASSIFIED & 46 \\
\hline $\begin{array}{l}\text { 20. SECURITY CLASS } \\
\text { (THIS PAGE) }\end{array}$ & 22. PriCe \\
UNCL ASSIFIED & \\
\hline
\end{tabular}


